\title{
Analysis of the Input Noise Contribution in the Noise Temperature Measurements
}

\author{
Robert $\mathrm{Hu}$
}

\begin{abstract}
In measuring the noise temperature of a cryogenic microwave low-noise amplifier (LNA), the noise from the input thermal buffer, i.e., the coaxial cable that connects the noise source to the amplifier, needs to be correctly accounted for. With the amplifier's noise temperature approaching just a few Kelvins, the postulate used in calculating the cable's noise temperature, that the cable is homogeneous and has a linear temperature profile, commands a further inspection. This letter analyzes these assumptions and clarifies the situations in which they hold. To substantiate this Kelvin-level discretion, a LNA is designed and measured.
\end{abstract}

Index Terms-Noise temperature measurement.

\section{INTRODUCTION}

$\mathbf{M}$ ICROWAVE low-noise amplifier (LNA) is widely used in radio-astronomy receivers and its noise temperature can be just a few kelvins [1], [2]. To carry out accurate measurements both at room temperature and cryogenically, the noise from the input coaxial cable, which serves as a thermal buffer between the LNA and the noise source, needs to be known accurately. Ideally, by treating the cable as a uniform, i.e., homogeneous, medium with constant thermal conductivity, temperature along the cable can be assumed linear [3]. However, since a coaxial cable comprises inner conductor, outer conductor and dielectric insulator in between, and, even worse, thermal conductivity is in general a function of temperature, this linear assumption seems susceptible and should be reexamined. Knowledge of this input thermal buffer's noise will be even critical in the more demanding areas, such as cryogenic on-wafer noise measurements [4], [5].

In this letter, by modeling the coaxial cable as a two-port thermal transmission line, it is proved that the temperature of the inner conductor does follow that of the outer conductor, except at the very boundaries. So, with caution, linear temperature profile can be adopted and a close-form expression for the coaxial cable is therefore derived. If the cable is low-loss, a further simplification ensues.

\section{CABle’s Temperature Profile}

To obtain the temperature profile along the coaxial cable, a thermal model needs to be constructed first (see Figs. 1 and 2).

Manuscript received March 24, 2004; revised October 28, 2004. The review of this letter was arranged by Associate Editor A. Stelzer.

The author is with the Department of Electronics Engineering, National Chiao Tung University (NCTU), Hsinchu, Taiwan, R.O.C.

Digital Object Identifier 10.1109/LMWC.2004.842832

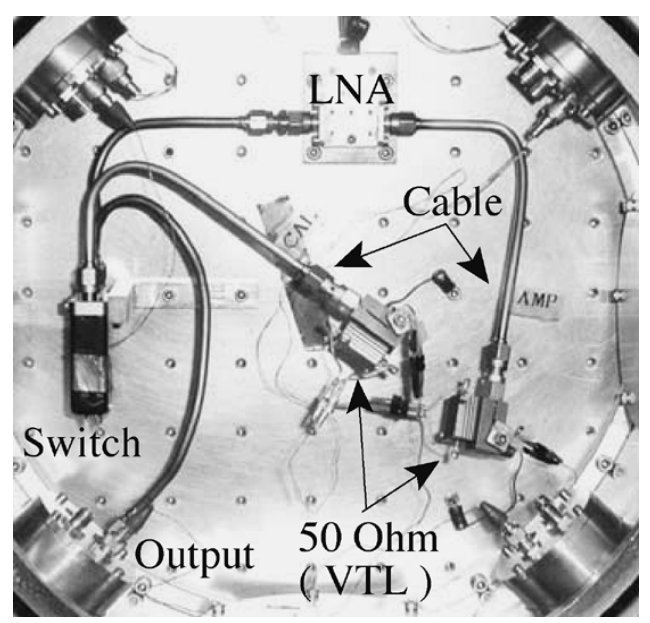

Fig. 1. Cryogenic noise-temperature measurement using variable-temperature load method. The temperature of the $50-\Omega$ resistors, as indicated, can be changed and monitored during the measurement. The input 5-in coaxial cable on the LNA branch works as a thermal buffer to prevent the LNA from heating up by the variable-temperature $50-\Omega$ resistor. The other 5 -in coaxial cable, as directly connecting to the switch, is for calibration purpose.

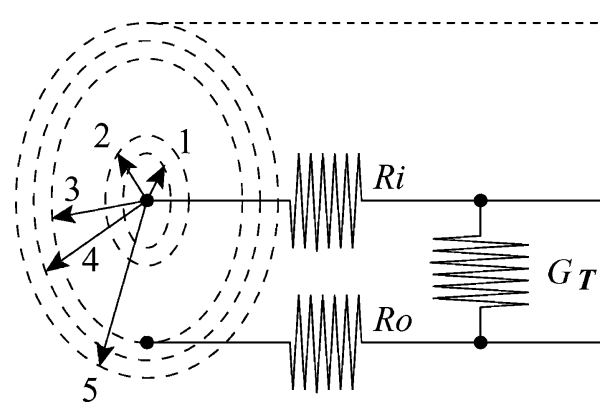

Fig. 2. Coaxial cable modeling. This distributed cable model comprises the inner and outer thermal resistance $R_{i}, R_{o}$, and the radial (shunt) thermal conductance $G_{T}$. Radii $r_{1}$ to $r_{5}$, as indicated by the arrowed lines, are the radii of the boundary of the inner stainless steel, inner copper, Teflon, optional outer copper, outer stainless steel and jacket, respectively.

The cable, as discussed throughout this letter, is the 5-in series- $A$ cable made by SSI Cable Corporation, which can be modeled thermally with

$$
\begin{array}{ll}
\frac{\partial T_{i}}{\partial x}=-I_{i} R_{i}, & \frac{\partial I_{i}}{\partial x}=-\left(T_{i}-T_{o}\right) G_{T} \\
\frac{\partial T_{o}}{\partial x}=-I_{o} R_{o}, & \frac{\partial I_{o}}{\partial x}=-\left(T_{o}-T_{i}\right) G_{T}
\end{array}
$$


where $T_{i}, T_{o}, I_{i}$, and $I_{o}$ are the temperatures and heat flows along the inner and outer conductors. The three constants $R_{i}$, $R_{o}$, and $G_{T}$ are linked to the cable's geometry

$$
\begin{aligned}
\frac{1}{R_{i}} & =\pi r_{1}^{2} K_{S}+\pi\left(r_{2}^{2}-r_{1}^{2}\right) K_{C} \\
\frac{1}{R_{o}} & =\pi\left(r_{4}^{2}-r_{3}^{2}\right) K_{C}+\pi\left(r_{5}^{2}-r_{4}^{2}\right) K_{S} \\
\frac{1}{G_{T}} & =\int_{r_{2}}^{r_{3}} \frac{d r}{2 \pi r K_{T}}=\frac{\ln \left(\frac{r_{3}}{r_{2}}\right)}{2 \pi K_{T}} .
\end{aligned}
$$

The thermal conductivity $K_{S}$ of the stainless steel at room temperature is, according to Goodfellow, around 16.3, pure copper has $K_{C}=401$, alloy copper such as $\mathrm{Cu}^{98} \mathrm{Be}^{2}$ has $K_{C}$ between 60 and 120, and Teflon has $K_{T}=0.25$, all with unit of $\mathrm{W} /(\mathrm{m} \cdot \mathrm{K})$. Therefore, this SSI coaxial cable has $R_{i}=$ $15.89 \cdot 10^{3}(\mathrm{~m} \cdot \mathrm{K}) / \mathrm{W}, R_{o}=19.55 \cdot 10^{3}(\mathrm{~m} \cdot \mathrm{K}) / \mathrm{W}$, and $G=1.3485 \mathrm{~W} /(\mathrm{m} \cdot \mathrm{K})$.

At first glance, since the shunt thermal conductance $G_{T}$ is large as compared with $R_{i}^{-1}$ and $R_{o}^{-1}$, the temperature difference between the inner and outer conductors on the same cross section should be very small except at the boundaries, which is defined by the characteristic distance $\alpha^{-1}$ with

$$
\alpha=\sqrt{\left(R_{i}+R_{o}\right) G_{T}}
$$

Mathematically, the solution of (1), i.e., the temperature distribution on the cable with length $L$, is

$$
\begin{aligned}
& T_{i}(x)=+A \frac{r_{i} G_{T} e^{\alpha x}}{\alpha^{2}}+B \frac{r_{i} G_{T} e^{-\alpha x}}{\alpha^{2}}+C x+D \\
& T_{o}(x)=-A \frac{r_{o} G_{T} e^{\alpha x}}{\alpha^{2}}-B \frac{r_{o} G_{T} e^{-\alpha x}}{\alpha^{2}}+C x+D .
\end{aligned}
$$

The four unknowns $A, B, C$, and $D$ can be easily decided from the four end-point constrains $T_{i 1}, T_{i 2}, T_{o 1}$, and $T_{o 2}$ on the inner and outer conductors. If the inner and outer conductors are thermally connected, i.e., $\alpha^{-1}=0$, then $T_{i 1}=T_{o 1}, T_{i 2}=T_{o 2}$, and their corresponding temperature profiles must be linear and identical. If $\alpha^{-1}=\infty$, such as air line, then the inner and outer temperature profiles are linear but unrelated.

As for the intended coaxial cable, its characteristic distance $\alpha^{-1}$ at room temperature is 0.18 in $(4.57 \mathrm{~mm})$ which is a small fraction of the total cable length; therefore, a linear temperature profile holds (Fig. 3). As temperature decreases, thermal conductivities of both stainless steel and Teflon decrease while that of copper will increase. The composite effects will cause the cable's cryogenic characteristic distance $\alpha^{-1}$ to increase but is still far less than the cable's total length. In short, the coaxial cable can be treated as a uniform medium in most cryogenic noise measurements.

Another implication of the simulated results is that, even the heater and temperature sensor in Fig. 1 are attached to the outside of the $50-\Omega$ termination, temperature inside the termination does follow that on the outside and the temperature sensor can record correctly.

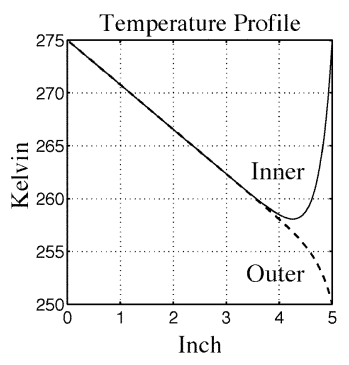

(a)

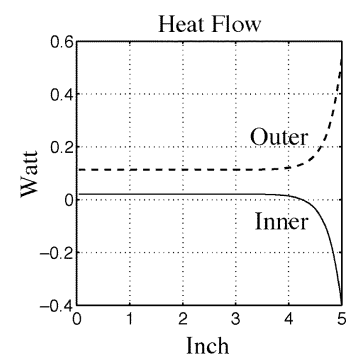

(b)
Fig. 3. Simulated temperature profile and power transfer along the 5-in coaxial cable. (a) The inner and outer temperature profiles. The four end-point temperatures of this cable are set to be $300,300,300$, and $250 \mathrm{~K}$. The large radial thermal conductivity of the cable means the temperature of the inner conductor is following that of the outer conductor on most part of the cable. (b) The corresponding inner and outer heat transfers. Most of the heat is flowing in the radial direction and is confined at one end.

\section{CABle's Noise Temperature Calculation}

For a two-port lumped passive component, that is reciprocal and has no input reflection, its noise temperature is $T_{\mathrm{amb}}(1-$ $G) / G$ where $G$ is the power gain, or $\left|S_{21}\right|^{2}$, and $T_{\mathrm{amb}}$ is the ambient temperature. By dividing the cable into $(m+1)$ infinitesimal sections where the $i$ th section has power gain $g_{i}=g=$ $G^{1 /(m+1)}$ and physical temperature $T_{i}$, the cable's noise temperature is

$$
\begin{aligned}
T_{\text {cable }} & =\lim _{m \rightarrow \infty} \frac{\sum_{i=0}^{m} T_{i}(1-g) g^{m-i}}{G} \\
& =\lim _{m \rightarrow \infty}\left[\frac{T_{A}(1-G)}{G}+\frac{T_{B}-T_{A}}{G}\left(1+\frac{G-g}{1-g} \frac{1}{m}\right)\right]
\end{aligned}
$$

where $T_{A}$ and $T_{B}$ are the end-point temperatures and a linear temperature profile along the cable is assumed. Of course, if $T_{A}=T_{B}$, then $T_{\text {cable }}=T_{A}(1-G) / G$. Otherwise, since

$$
g=G^{\frac{1}{(m+1)}}=1+\left[\frac{\ln G}{m+1}\right]+\left[\frac{\ln G}{m+1}\right]^{2}+\cdots
$$

or

$$
\lim _{m \rightarrow \infty}(1-g) m=-\ln G
$$

there is

$$
T_{\text {cable }}=\frac{1}{G}\left[T_{A}(1-G)+\left(T_{B}-T_{A}\right)\left(1+\frac{1-G}{\ln G}\right)\right] .
$$

If the cable is low-loss, i.e., $\mathrm{G}=1-\rho$ and $0 \leq \rho \ll 1$, then, as $\ln G \approx-\rho-\rho^{2} / 2$, the above expression can be simplified as

$$
\begin{aligned}
T_{\text {cable }} & =\frac{1}{G} \frac{2 \rho T_{A}+\rho^{2} T_{A}+\rho T_{B}-\rho T_{A}}{2+\rho} \\
& =\frac{1-G}{G} \frac{T_{A}+T_{B}}{2} .
\end{aligned}
$$

In this situation, the cable can be treated as a lumped element with equivalent physical temperature of $\left(T_{A}+T_{B}\right) / 2$.

However, since thermal conductivity is in general a function of temperature rather than a constant, the real temperature profile along the cable is more complicated. As an example, in the cryogenic noise temperature measurement in the liquid Helium (LHe) cryostat where one end of the coaxial cable is heated from 4 to $20 \mathrm{~K}$ while the other end is kept at $4 \mathrm{~K}$, a copper cable with 


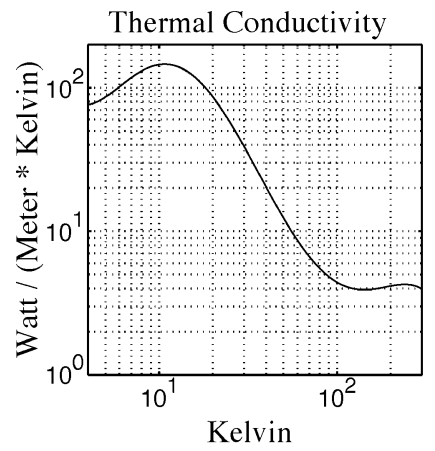

(a)

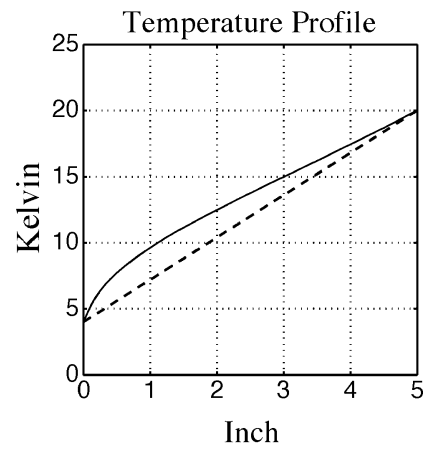

(b)
Fig. 4. Temperature profile of the coaxial cable used in the cryogenic noisetemperature measurements. (a) The assumed thermal conductivity of pure copper from 4 to $300 \mathrm{~K}$. (b) The temperature profile along the 5-in cable made with copper that has end-point temperatures of 4 and $20 \mathrm{~K}$. The solid curve is the simulated result while the dashed curve is its linear counterpart.

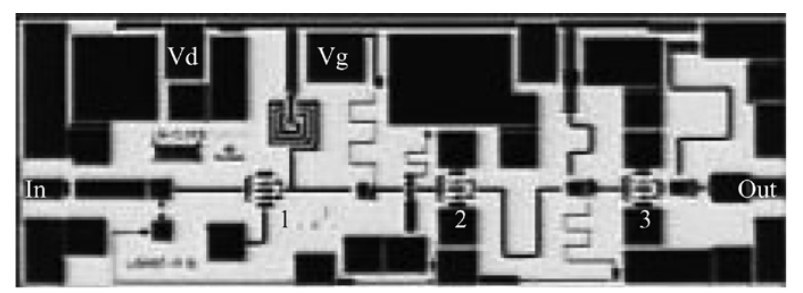

(a)

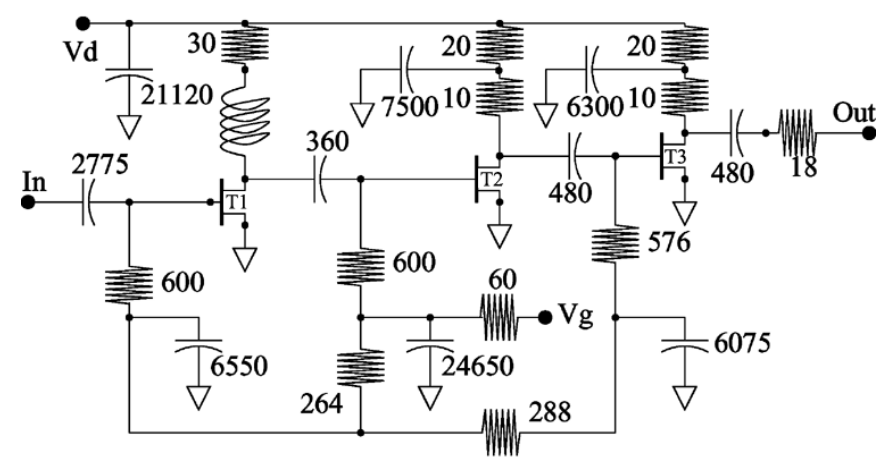

(b)

Fig. 5. LNA circuit. (a) This is a three-stage amplifier with common drain and gate biases, and is fabricated by Raytheon. The layout has dimensions of $2000 \times 750 \times 75 \mu \mathrm{m}^{3}$. (b) In the schematic, the capacitance is in fentofarads and the resistance is in $\Omega$.

$0.5-\mathrm{dB}$ loss should have, through simulation, equivalent physical temperature of $13.5 \mathrm{~K}$ rather than from $(20+4) / 2=12 \mathrm{~K}$ (Fig. 4). Nonetheless, the cable's output noise-temperature difference, which is directly related to the measured noise-temperature uncertainty of the LNA, is a mere $\Delta T(1-G) / G=$ $1.5 \cdot(1-0.89) / 0.89=0.185 \mathrm{~K}$. In other words, except in the ultra-low-noise cryogenic LNA measurements or in the more demanding noise-parameter measurements, the use of the above noise expression is acceptable. As an illustration, a 10-K cryogenic amplifier is designed and tested (Figs. 5 and 6), where the aforementioned $0.185 \mathrm{~K}$ offset appears to be tolerable.

So far, the other noise-temperature measurement method, the so-called cold-attenuator method where the input coaxial cable is connecting the commercial noise source at room temperature to the cold attenuator inside the cryostat [6], is left undiscussed. This is because, from the above analysis, it is obvious that the

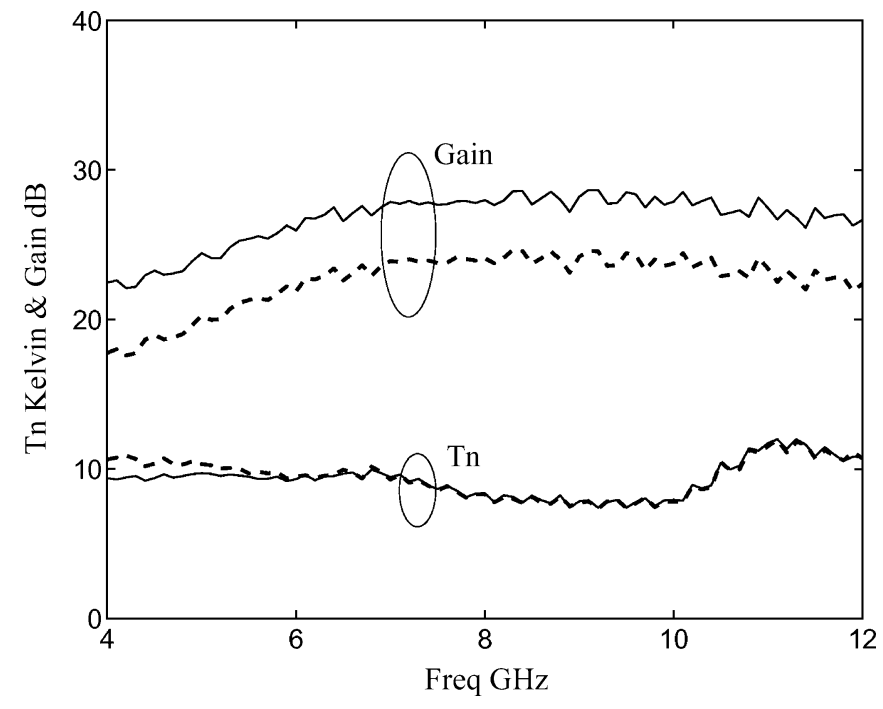

Fig. 6. Noise temperature $T_{n}$ and gain of the LNA inside the $4 \mathrm{~K}$ liquid Helium dewar. The solid curves correspond to bias condition of $V_{d}=0.8 \mathrm{~V}$ and $I_{d}=$ $23 \mathrm{~mA}$ while the dashed curves are with $V_{d}=0.5 \mathrm{~V}$ and $I_{d}=10 \mathrm{~mA}$.

cable's noise contribution cannot be accurately retrieved. And quite often, the resulting noise temperature of a sensitive amplifier measured using the cold-attenuator method is negative, which apparently is due to an over-estimation of the cable's noise. It is too bold assuming the equivalent physical temperature of a cable stretching from room temperature (around $296 \mathrm{~K}$ ) to $4 \mathrm{~K}$ is simply $(296+4) / 2=150 \mathrm{~K}$. Practically, cold-attenuator method needs to be calibrated with the help of variable-temperature-load method.

\section{CONCLUSION}

In this letter, the noise of the input coaxial cable used in the noise-temperature measurements has been analyzed, and the condition where close-form expressions hold is clarified. As an illustration, a cryogenic amplifier is designed and tested. Its low noise temperature justifies this letter's kelvin-level discretion.

\section{ACKNOWLEDGMENT}

The author wishes to thank Dr. S. Weinreb and T. Vayonakis, Caltech, and J. Ward, JPL, for their suggestions.

\section{REFERENCES}

[1] S. Weinreb, D. L. Fensternmacher, and R. W. Harris, "Ultra-low-noise 1.2- to 1.7-GHz cooled GaAsFET amplifier," IEEE Trans. Microw. Theory Tech., vol. 82, no. 6, pp. 847-853, Jun. 1982.

[2] N. Wadefalk et al., "Cryogenic wide-band ultra-low-noise IF amplifiers operating at ultra-low DC power," IEEE Trans. Microw. Theory Tech., vol. 51, no. 6 , pp. 1705-1711, Jun. 2003.

[3] J. Ward, "Observation of Carbon Monoxide in the Starburst Galaxy M82 With a $690 \mathrm{GHz}$ Wide Receiver," Ph.D. dissertation, California Inst. Technol., Pasadena, CA, 2002.

[4] J. Laskar, J. J. Bautista, M. Nishimoto, M. Hamai, and R. Lai, “Development of accurate on-wafer, cryogenic characterization technique," IEEE Trans. Microw. Theory Tech., vol. 44, no. 7, pp. 1178-1183, Jul. 1996.

[5] A. Caddemi and N. Donato, "Characterization techniques for temperature-dependent experimental analysis of microwave transistor," IEEE Trans. Instrum. Meas., vol. 52, no. 1, pp. 85-91, Feb. 2003.

[6] J. D. Gallego, "Definition of measurements of performance of X band cryogenic amplifiers," Technical Note ESA/CAY-01 TN01, Centro Astronomico de yebes Observatorio Astronomico Nacional, Madrid, Spain, 2000 . 